\title{
Added value of gastrin receptor scintigraphy in comparison to somatostatin receptor scintigraphy in patients with carcinoids and other neuroendocrine tumours
}

\author{
Martin Gotthardt, Martin P Béhé ${ }^{1}$, Julia Grass ${ }^{1}$, Artur Bauhofer ${ }^{2}$, Anja Rinke ${ }^{3}$, \\ Meike L Schipper ${ }^{1}$, Marc Kalinowski ${ }^{4}$, Rudolf Arnold ${ }^{4}$, Wim J G Oyen and \\ Thomas M Behr ${ }^{1}$
}

Department of Nuclear Medicine, Radboud University Nijmegen Medical Center, Postbus 9101, 6500 HB Nijmegen, The Netherlands ${ }^{1}$ Department of Nuclear Medicine, ${ }^{2}$ Institute of Theoretical Surgery, ${ }^{3}$ Departments of Gastroenterology and Endocrinology and

${ }^{4}$ Diagnostic Radiology, Philipps-University of Marburg, Baldingerstrasse, 35043 Marburg, Germany

(Requests for offprints should be addressed to M Gotthardt; Email: m.gotthardt@nucmed.umcn.nl)

\begin{abstract}
Gastrin receptor scintigraphy (GRS) is a new imaging method primarily developed for the detection of metastases of medullary thyroid carcinoma (MTC). As gastrin-binding $\mathrm{CCK}_{2}$ receptors are also expressed on a variety of other neuroendocrine tumours (NET), we compared GRS to somatostatin receptor scintigraphy (SRS) in patients with NET. SRS and GRS were performed within 21 days in a series of 60 consecutive patients with NET. GRS was directly compared with SRS. If lesions were visible on GRS but not detectable by SRS, other imaging modalities (MRI, CT) and follow-up were used for verification. Of the 60 evaluable patients, 51 had carcinoid tumours, 3 gastrinomas, 2 glucagonomas, 1 insulinoma and 3 paragangliomas. The overall tumour-detection rate was $73.7 \%$ for GRS and $82.1 \%$ for SRS. In the 11 patients with negative SRS, GRS was positive in $6(54.5 \%)$. Based on the number of tumour sites detected and the degree of uptake, GRS performed better than SRS in 13 patients $(21.7 \%)$, equivalent images were obtained in 18 cases $(30.0 \%)$ and SRS performed better in $24(40.0 \%)$ cases. In six of the SRS positive patients, 18 additional sites of tumour involvement could be detected. Overall, GRS detected additional tumour sites in $20 \%$ of the patients. Localisation of the primary tumours or their functional status had no influence on the outcome of imaging. GRS should be performed in selected patients as it may provide additional information in patients with NET with equivocal or absent somatostatin uptake.
\end{abstract}

Endocrine-Related Cancer (2006) 13 1203-1211

\section{Introduction}

Somatostatin receptor scintigraphy (SRS) is a successful nuclear medicine procedure for the detection of neuroendocrine tumours (NET) and their metastases. It is considered as the diagnostic method of choice in several tumour entities (Krenning et al. 1993, Modlin \& Tang 1997, Ricke et al. 2001). The method is based on specific binding of radiopeptides mainly to the somatostatin receptor subtype 2 (sstr2) overexpressed by tumour cells (Lamberts et al. 1990). Residualising labels have been introduced to improve image quality (Bakker et al. 1991). In radiopeptide scintigraphy, these labels usually consist of radiometal-chelator complexes bound to the respective peptides. After specific binding and internalisation, the complexes are retained inside the cell (metabolic trapping) while activity in blood and other tissues decreases. This results in high tumour-to-background ratios. 
Radiopeptide imaging has a considerable impact on patient management in the range of $25-50 \%$ (Lebtahi et al. 1997, Termanini et al. 1997). However, some patients show low or even absent uptake in SRS. Especially, patients with medullary thyroid carcinoma (MTC) are often sstr2-negative. As recurrent MTC can chemically be diagnosed by pentagastrin stimulation testing, a radiopeptide has been developed for targeting the pentagastrin-binding $\mathrm{CCK}_{2}$ receptor. This peptide has shown first promising results in imaging of recurrent and occult MTC (Behr et al. 1999a,b, Gotthardt et al. 2006).

The $\mathrm{CCK}_{2}$ receptor is also expressed in other NET, such as gastrointestinal NET (Reubi \& Waser 2003). Therefore, the use of a $\mathrm{CCK}_{2}$ receptor-binding compound may provide additional diagnostic information. We compared the results of SRS and gastrin receptor scintigraphy (GRS) in a series of patients with neuroendocrine tumours.

\section{Materials and methods}

\section{Patients and study design}

We included a series of consecutive patients who underwent staging for histologically verified NETs between July 2001 and July 2003 at the university hospital in Marburg. All patients underwent GRS and SRS within 21 days to guarantee for comparability. All patients had given written consent for participation in the study. The study was approved by the local ethics committee and the radiation protection authorities.

\section{Scintigraphic imaging protocol}

\section{Somatostatin receptor scanning and gastrin receptor scanning}

Four and $24 \mathrm{~h}$ after injection of $150-200 \mathrm{MBq}$ ${ }^{111}$ In-DPhe ${ }^{1}$-DTPA-Octreotide (OctreoScan, Tyco Healthcare, Neustadt/Donau, Germany) or ${ }^{111}$ InDTPA-DGlu ${ }^{1}$-minigastrin (DTPA-DGlu ${ }^{1}$-minigastrin synthesised by Bachem, Weil am Rhein, Germany), anterior and posterior planar whole body images were obtained. A Siemens dual-head gamma camera (E CAM, Siemens, Hofman Estates, IL, USA) was used, equipped with medium energy parallel hole collimators. The scanning speed was set to $7 \mathrm{~cm} / \mathrm{min}$. For imaging, the compound was labelled with a specific activity of $55 \mathrm{GBq} / \mu \mathrm{mol}$, the radiochemical purity of the labelled minigastrin was always $>95 \%$ (HPLC) as described previously (Behe et al. 2005, Behr et al. 1999a,b).

\section{Other imaging techniques}

\section{Computed tomography}

A Siemens Somatom 2 scanner was used. Using an automated power injector $(70 / 120 \mathrm{ml}$ volume, flow rate 2-3 ml/s), non-ionic contrast medium (Iopamidol 300; Solutrast, Bracco-Byc Gulden, Konstanz, Germany) was administered intravenously. Slices of $3-8 \mathrm{~mm}$ were obtained at 4.5 or $12 \mathrm{~mm}$ increments from scull base to jugulum and from lung apex to pubic symphysis.

\section{Magnetic resonance imaging}

Scans were acquired using a $1.0 \mathrm{~T}$ scanner (Magnetom Expert, Siemens, Erlangen, Germany) with commercially available gradients capable of a $1200 \mu$ s rise time and $20 \mathrm{mT} / \mathrm{m}$ maximum gradient strength using a body array coil. A T2 weighted axial turbo spin echo sequence (TR/TE 2730/138 ms, SL $6 \mathrm{~mm}$ ) and an axial T1 weighted fast low angle shot gradient sequence (TR/TE 134/6 ms, FA 70 ${ }^{\circ}$, SL6) were used prior to and after injection of the contrast agent (Gd-DTPA (Magnevist), $0.1 \mathrm{mmol} / \mathrm{kg}$, flow of $1 \mathrm{ml} / \mathrm{s}$ ), and a coronal true fast imaging with steady-state precession sequence (TR/TE 10.22/4.7 ms, SL $5 \mathrm{~mm}$ ).

\section{Evaluation of SRS and GRS}

SRS and GRS images were evaluated independently by two observers who were experienced specialists in nuclear medicine. The observers were blinded with respect to patient identity, extent of disease and results of other imaging procedures, only the tumour type was known. In case of discordance, the differences were discussed together and settled (consensus reading).

As patient management depends more on involvement of a region (e.g. left liver lobe resection if right liver lobe is not involved) than on the total number of metastases, a regional assessment of tumour localisations was performed (cervical region, lung, supraclavicular, upper and lower limbs, liver, pelvis (all specified by involved side), peritoneal, retroperitoneal, cervical/thoracic/lumbar spine, mediastinal, mesenterial, pancreas and skull).

GRS was directly compared with SRS as it is the established standard technique for scintigraphic imaging of NETs. If foci were negative on SRS but positive on GRS, CT and MRI as described previously as well as follow-up were used for verification of these findings according to the method described by Juweid et al. (1996). Using these imaging modalities, foci had to be depicted in a typical manner to be rated as true-positive findings. As GRS would be underscored if it is truepositive while SRS is false-negative, it is necessary to verify such lesions by other techniques. As histologic evaluation of all detected lesions is not ethical, this is the 
best approach to the problem of verification of findings false-positive in the standard of comparison.

Octreotide uptake was assessed in comparison with liver uptake using a modification of the score of Krenning et al. (1993) (1, lower than; 2, equivalent to and 3, higher than liver). The gastrin uptake was visually evaluated in comparison with SRS as 1, lower; 2 , equivalent or 3 , higher. If SRS was negative, gastrin uptake was determined in comparison with the thyroid uptake in analogy to the score described previously. For both SRS and GRS, the average uptake was calculated. The final score was derived from multiplication of the average tumour uptake value and the number of tumour sites detected.

Ki67 as a marker of tumour proliferation was documented as high $(>10 \%)$, intermediate $(2-10 \%)$ or low $(<2 \%)$. Ki67 and the grading (high/intermediate/low differentiation) of the tumour as determined by histology were used for the determination of a possible correlation to the outcome of GRS and SRS (GRS better, SRS better or SRS and GRS equal).

\section{Statistical analysis}

Descriptive statistics were used giving a tumourdetection rate for comparison of SRS with GRS. As only histological evaluation of all lesions would allow adequate calculation of specificity of an imaging method, specificity has not been determined. $\kappa$-Statistics were used to evaluate the inter-rater reliability for gastrin and somatostatin scanning. The correlation of the results of scintigraphy with Ki67 expression and grading was determined by Pearson's correlation coefficient. Correlation between outcome of scintigraphy and functional status/localisation of the tumours was determined in the same manner (paragangliomas were excluded from this evaluation).

\section{Results}

\section{Patients}

From July 2001 to July 2003, 122 GRS and SRS scans of 61 consecutive patients with NETs (27 female, 33 male; mean age $47.7 \pm 11.4$ years) were eligible for the study. GRS and SRS scans performed during follow-up of these 60 patients or scans not performed within the time limit of 21 days were not analysed. One patient thought to have a pulmonary carcinoid that proved to be small cell lung cancer later was excluded from the study.

Of the 60 evaluable patients, 51 had carcinoid tumours, 3 gastrinomas, 2 glucagonomas, 1 insulinoma and 3 paragangliomas. The majority of patients

Table 1 Comparison of GRS and SRS for all patients and for the subgroups

No. of patients

Percentage of patients

Comparison of GRS/SRS in all patients with NET, 224 tumour sites

No. of lesions detected: GRS 165; SRS 184

Tumour detection rate: GRS $73.7 \%$; SRS $82.1 \%$

GRS $>$ SRS

GRS $=$ SRS

GRS $<$ SRS

GRS- and SRS-negative

SRS-negative

GRS-positive from SRS-negative

Comparison of GRS/SRS in patients with carcinoids

No. of lesions detected: GRS 159; SRS 173

Tumour detection rate: GRS $78.2 \%$; SRS $85.2 \%$

GRS $>$ SRS

$\mathrm{GRS}=\mathrm{SRS}$

GRS $<$ SRS

SRS-negative

GRS-positive from SRS-negative

13

18

24

5

11

$6 / 11$

Comparison of GRS/SRS in patients with functional pancreatic NET

No. of lesions detected: GRS 5; SRS 4

Tumour detection rate: GRS $71.4 \%$; SRS $57.1 \%$

GRS $>$ SRS

$\mathrm{GRS}=\mathrm{SRS}$

GRS $<$ SRS

GRS- and SRS-negative

GRS-positive and SRS-negative

GRS-positive from SRS-negative
21.7

30.0

40.0

8.3

18.3

54.5 
$(n=54)$ had known metastatic disease, four had recurrent disease after $\mathrm{R}_{0}$ resection of the primary, one had local recurrence after $R_{1}$ resection, and one had no known metastases after $\mathrm{R}_{0}$ resection.

Side effects after the injection of minigastrin matched with those of pentagastrin testing (i.e. nausea, flush, tickling and hypotension) and were generally mild. In two patients, more severe side effects were observed (decrease in blood pressure to levels of approximately 80 over $50 \mathrm{mmHg}$ ), both recovering quickly without intervention.

(a)

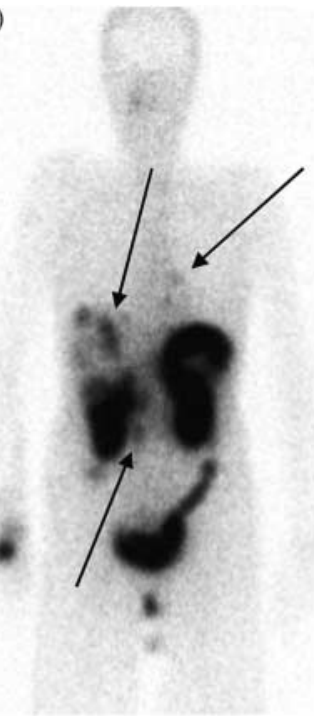

(c)

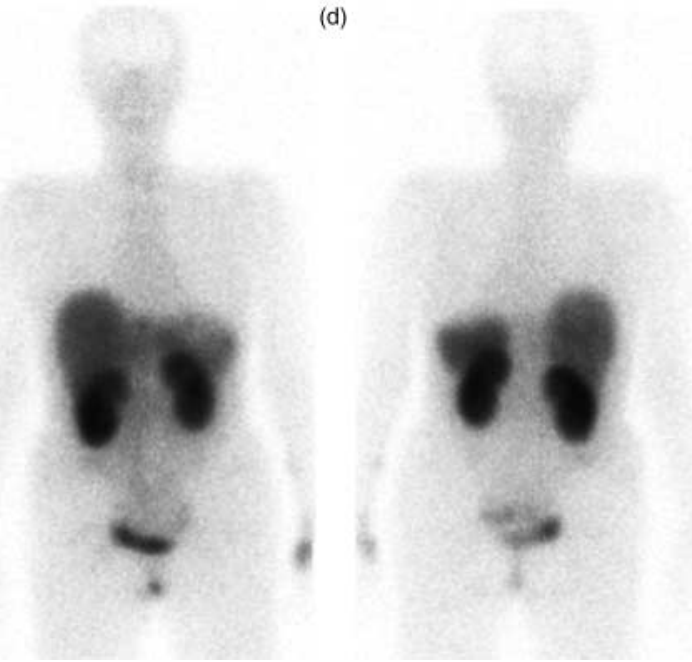

(b)

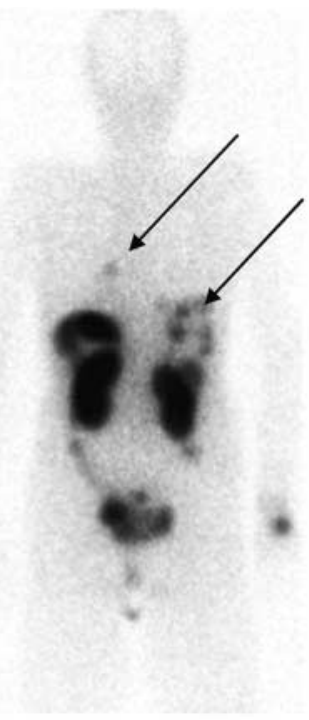

(d)

Figure 1 Patient with a carcinoid of the smaller intestine, metastases to liver, mediastinum and paraaortic lymph nodes. High uptake in the paraaortic and liver lesions can be seen on GRS (a), anterior and (b), posterior view. On SRS (c), anterior and (d), posterior view), the large lesion in the liver is barely visible. (a)

(b)
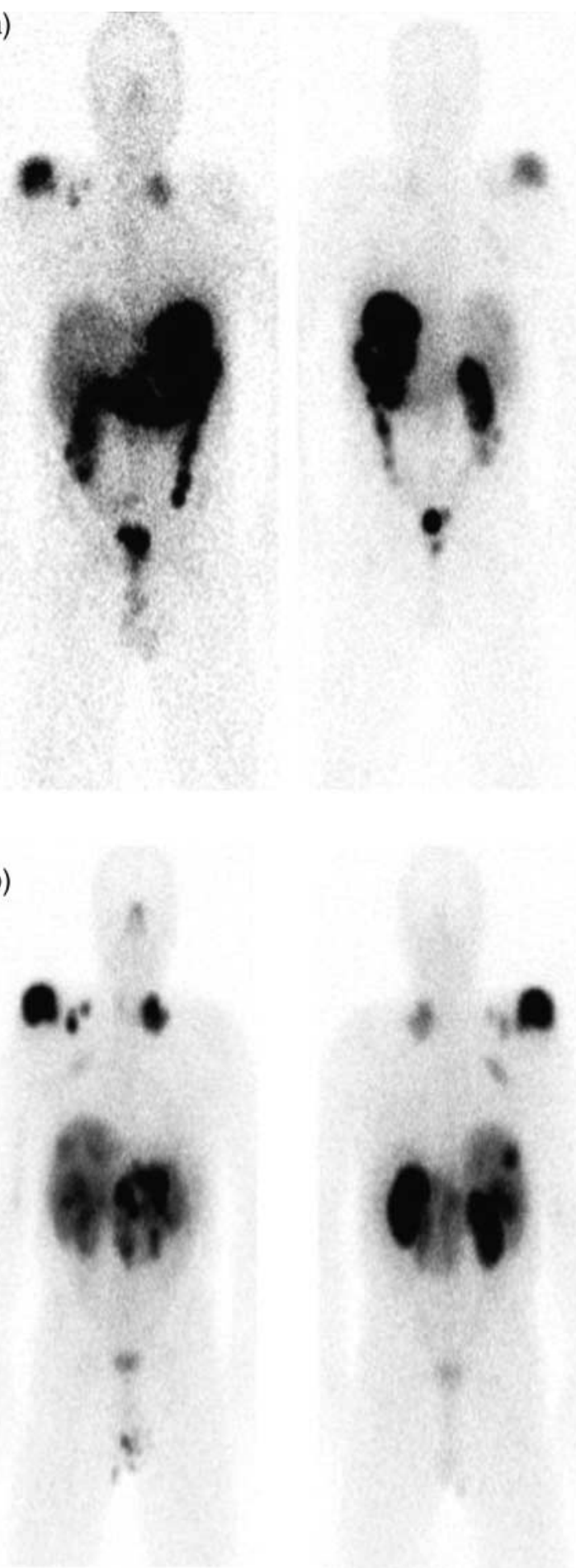

Figure 2 Patient with a metastasised carcinoid of the pancreatic tail. GRS (a) shows relatively low uptake into the bone metastases in the shoulder girdle and no uptake in the liver metastases. In contrast, SRS is strongly positive in all lesions (b); all scans are $24 \mathrm{~h}$ scans, anterior view left, posterior view right. 
(a)

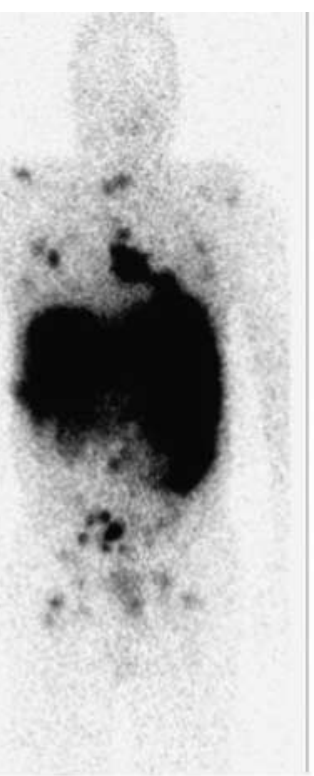

(b)

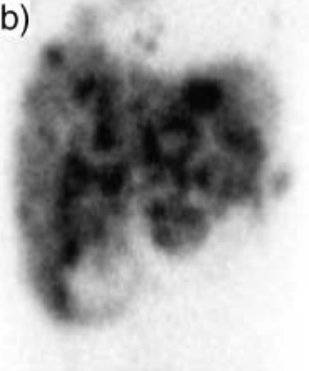

(c)

Figure 3 Patient with a bronchial carcinoid with metastases to lung, lymph nodes, liver and bone. GRS is positive (a), including uptake in liver metastases; (b) shows a differently windowed detail image of the liver. SRS is negative (c); all scans are $24 \mathrm{~h}$ scans, anterior view left, posterior view right.

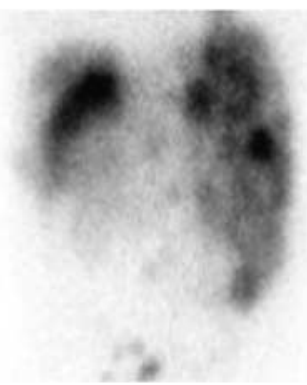

\section{Comparison of somatostatin and gastrin scans}

The total number of involved sites was 224. GRS detected 165 and SRS 184 . The overall tumour-detection rate was thus $73.7 \%$ for GRS and $82.1 \%$ for SRS. In 11 patients with negative SRS, GRS was positive in 6 (54.5\% of SRS-negative patients). In 6 of the SRSpositive patients (12.2\% of 49 SRS-positive patients), GRS was able to detect 18 additional sites of tumour involvement. Therefore, GRS detected tumour sites missed by SRS in 12 patients (20\%). Average tumour uptake values were $1.8 \pm 1.1$ in SRS and $1.5 \pm 1.0$ in

(a)
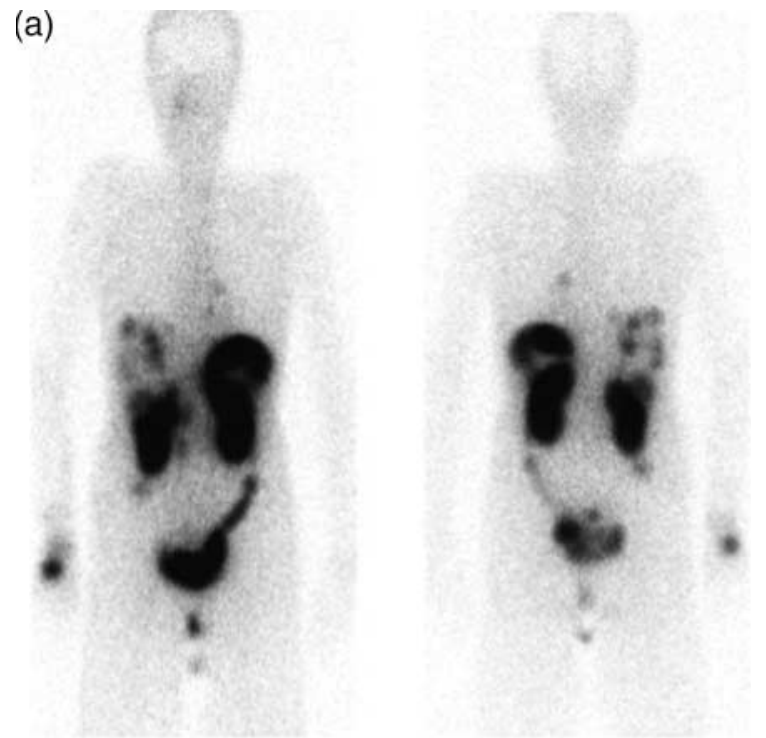

(b)

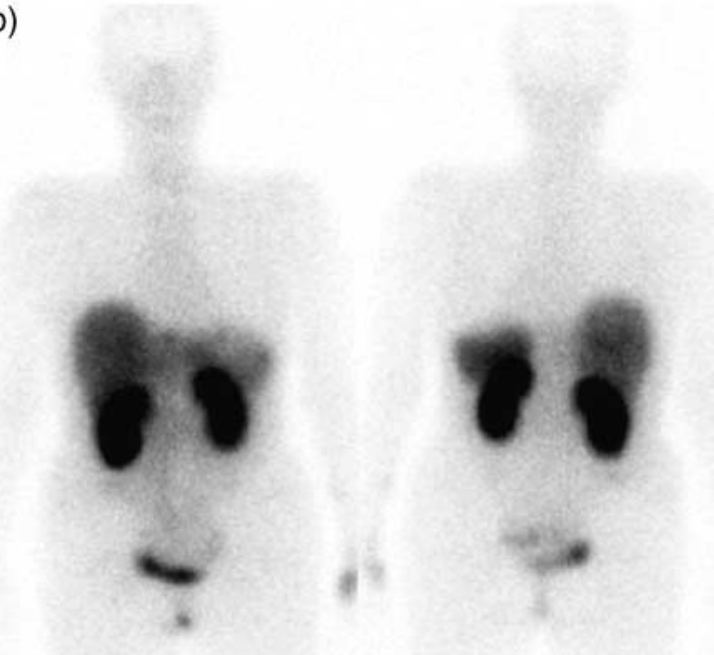

Figure 4 Patient with a metastasised carcinoid of the ileum. While GRS is positive (a) showing lesions in the liver and the left mediastinum, SRS is negative (b); all scans are $24 \mathrm{~h}$ scans, anterior view left, posterior view right). 
Table 2 Patient characteristics and per patient analysis of imaging

\begin{tabular}{|c|c|c|c|c|c|c|}
\hline No. & Age & Sex & Diagnosis & Receptor status & Result & Active \\
\hline 1 & 55 & $f$ & Carcinoid (mg) & S-pos./G-neg. & $S>G$ & $y$ \\
\hline 2 & 65 & $\mathrm{~m}$ & Carcinoid (fg) & S-pos./G-pos. & $\mathrm{S}=\mathrm{G}$ & $\mathrm{n}$ \\
\hline 3 & 37 & $\mathrm{~m}$ & Carcinoid (fg) & S-pos./G-pos. & $S>G$ & $\mathrm{n}$ \\
\hline 4 & 33 & $f$ & Paraganglioma & S-pos./G-neg. & $S>G$ & \\
\hline 5 & 47 & $\mathrm{~m}$ & Gastrinoma & S-neg./G-neg. & Negative & y \\
\hline 6 & 38 & $\mathrm{~m}$ & Carcinoid (fg) & S-pos./G-pos. & $\mathrm{S}<\mathrm{G}$ & y \\
\hline 7 & 51 & $f$ & Carcinoid (mg) & S-pos./G-pos. & $S=G$ & y \\
\hline 8 & 30 & $f$ & Gastrinoma & S-neg./G-neg. & Negative & y \\
\hline 9 & 24 & $f$ & Carcinoid (fg) & S-pos./G-pos. & $S>G$ & y \\
\hline 10 & 50 & $f$ & Carcinoid (mg) & S-pos./G-pos. & $S=G$ & $\mathrm{n}$ \\
\hline 11 & 37 & $\mathrm{~m}$ & Paraganglioma & S-pos./G-pos. & $S>G$ & \\
\hline 12 & 42 & $f$ & Carcinoid (fg) & S-pos./G-pos. & $S>G$ & $\mathrm{n}$ \\
\hline 13 & 46 & $\mathrm{~m}$ & Carcinoid (fg) & S-pos./G-pos. & $\mathrm{S}<\mathrm{G}$ & $\mathrm{n}$ \\
\hline 14 & 28 & $f$ & Carcinoid (fg) & S-pos./G-pos. & $\mathrm{S}<\mathrm{G}$ & y \\
\hline 15 & 17 & $f$ & Carcinoid (fg) & S-pos./G-pos. & $\mathrm{S}=\mathrm{G}$ & $n$ \\
\hline 16 & 70 & $f$ & Carcinoid (fg) & S-pos./G-pos. & $S>G$ & $\mathrm{n}$ \\
\hline 17 & 49 & $\mathrm{~m}$ & Glucagonoma & S-neg./G-pos. & $\mathrm{S}<\mathrm{G}$ & y \\
\hline 18 & 68 & $\mathrm{~m}$ & Carcinoid (unclear) & S-pos./G-pos. & $S>G$ & $\mathrm{n}$ \\
\hline 19 & 50 & $f$ & Carcinoid (mg) & S-pos./G-pos. & $S>G$ & $y$ \\
\hline 20 & 57 & $f$ & Carcinoid (fg) & S-pos./G-pos. & $S>G$ & $\mathrm{n}$ \\
\hline 21 & 41 & $\mathrm{~m}$ & Carcinoid (fg) & S-pos./G-pos. & $\mathrm{S}=\mathrm{G}$ & $\mathrm{n}$ \\
\hline 22 & 39 & $f$ & Carcinoid (unclear) & S-pos./G-pos. & $S>G$ & $\mathrm{n}$ \\
\hline 23 & 59 & $\mathrm{~m}$ & Carcinoid (unclear) & S-pos./G-pos. & $\mathrm{S}<\mathrm{G}$ & $\mathrm{n}$ \\
\hline 24 & 49 & $f$ & Carcinoid (fg) & S-pos./G-pos. & $S=G$ & $\mathrm{n}$ \\
\hline 25 & 38 & $\mathrm{~m}$ & Carcinoid (mg) & S-pos./G-pos. & $S>G$ & $\mathrm{n}$ \\
\hline 26 & 39 & $f$ & Carcinoid (hg) & S-pos./G-neg. & $S>G$ & $\mathrm{n}$ \\
\hline 27 & 39 & $\mathrm{~m}$ & Carcinoid (fg) & S-pos./G-pos. & $S>G$ & $\mathrm{n}$ \\
\hline 28 & 66 & $\mathrm{~m}$ & Carcinoid (mg) & S-pos./G-pos. & $\mathrm{S}=\mathrm{G}$ & $\mathrm{y}$ \\
\hline 29 & 41 & $f$ & Carcinoid (mg) & S-neg./G-pos. & $\mathrm{S}<\mathrm{G}$ & $\mathrm{n}$ \\
\hline 30 & 40 & $\mathrm{~m}$ & Carcinoid (mg) & S-pos./G-pos. & $S>G$ & $\mathrm{n}$ \\
\hline 31 & 56 & $f$ & Carcinoid (mg) & S-pos./G-neg. & $S>G$ & $y$ \\
\hline 32 & 56 & $\mathrm{~m}$ & Carcinoid (mg) & S-pos./G-neg. & $S>G$ & y \\
\hline 33 & 49 & $f$ & Carcinoid (fg) & S-pos./G-pos. & $\mathrm{S}=\mathrm{G}$ & $\mathrm{n}$ \\
\hline 34 & 56 & $f$ & Carcinoid (mg) & S-neg./G-neg. & Negative & $y$ \\
\hline 35 & 61 & $\mathrm{~m}$ & Carcinoid (mg) & S-pos./G-pos. & $\mathrm{S}<\mathrm{G}$ & y \\
\hline 36 & 38 & $f$ & Carcinoid (mg) & S-pos./G-pos. & $\mathrm{S}<\mathrm{G}$ & $y$ \\
\hline 37 & 50 & $\mathrm{~m}$ & Carcinoid (mg) & S-pos./G-pos. & $\mathrm{S}=\mathrm{G}$ & $\mathrm{n}$ \\
\hline 38 & 50 & $f$ & Carcinoid (unclear) & S-pos./G-pos. & $\mathrm{S}=\mathrm{G}$ & y \\
\hline 39 & 48 & $\mathrm{~m}$ & Gastrinoma (MEN I) & S-pos./G-neg. & $S>G$ & y \\
\hline 40 & 53 & $\mathrm{~m}$ & Carcinoid (fg) & S-pos./G-pos. & $\mathrm{S}=\mathrm{G}$ & $\mathrm{n}$ \\
\hline 41 & 53 & $\mathrm{~m}$ & Carcinoid (mg) & S-pos./G-pos. & $\mathrm{S}=\mathrm{G}$ & y \\
\hline 42 & 43 & $f$ & Carcinoid (fg) & S-pos./G-pos. & $\mathrm{S}<\mathrm{G}$ & $\mathrm{n}$ \\
\hline 43 & 40 & $\mathrm{~m}$ & Carcinoid (fg) & S-neg./G-pos. & $\mathrm{S}<\mathrm{G}$ & $\mathrm{n}$ \\
\hline 44 & 25 & $\mathrm{~m}$ & Carcinoid (mg) & S-pos./G-pos. & $S>G$ & y \\
\hline 45 & 49 & $\mathrm{~m}$ & Carcinoid (mg) & S-neg./G-pos. & $\mathrm{S}<\mathrm{G}$ & y \\
\hline 46 & 55 & $f$ & Paraganglioma & S-pos./G-neg. & $S>G$ & \\
\hline 47 & 42 & $\mathrm{~m}$ & Carcinoid (fg) & S-pos./G-pos. & $S>G$ & $\mathrm{n}$ \\
\hline 48 & 55 & $\mathrm{~m}$ & Carcinoid (unclear) & S-pos./G-pos. & $\mathrm{S}=\mathrm{G}$ & y \\
\hline 49 & 62 & $\mathrm{~m}$ & Carcinoid (mg) & S-pos./G-pos. & $S>G$ & y \\
\hline 50 & 63 & $f$ & Carcinoid (hg) & S-neg./G-neg. & Negative & $\mathrm{n}$ \\
\hline 51 & 49 & $f$ & Carcinoid (fg) & S-pos./G-neg. & $\mathrm{S}>\mathrm{G}$ & $n$ \\
\hline 52 & 31 & $f$ & Glucagonoma & S-pos./G-pos. & $\mathrm{S}=\mathrm{G}$ & y \\
\hline 53 & 62 & $\mathrm{~m}$ & Carcinoid (fg) & S-pos./G-pos. & $S>G$ & $n$ \\
\hline 54 & 50 & $f$ & Carcinoid (fg) & S-neg./G-pos. & $\mathrm{S}<\mathrm{G}$ & $\mathrm{n}$ \\
\hline 55 & 47 & $\mathrm{~m}$ & Carcinoid (fg) & S-pos./G-pos. & $\mathrm{S}=\mathrm{G}$ & $\mathrm{n}$ \\
\hline 56 & 60 & $\mathrm{~m}$ & Carcinoid (mg) & S-pos./G-pos. & $S=G$ & $\mathrm{y}$ \\
\hline 57 & 48 & $f$ & Carcinoid (mg) & S-pos./G-pos. & $\mathrm{S}=\mathrm{G}$ & $\mathrm{n}$ \\
\hline 58 & 58 & $\mathrm{~m}$ & Carcinoid (unclear) & S-pos./G-pos. & $\mathrm{S}=\mathrm{G}$ & $y$ \\
\hline 59 & 52 & $\mathrm{~m}$ & Carcinoid (mg) & S-neg./G-neg. & Negative & y \\
\hline 60 & 55 & $f$ & Insulinoma & S-neg./G-pos. & $\mathrm{S}<\mathrm{G}$ & y \\
\hline
\end{tabular}

The localisation of the primary is characterised by $(\mathrm{hg})$ for hindgut, $(\mathrm{mg})$ for midgut, $(\mathrm{fg})$ for foregut and unclear if the localisation is not known. In the last column, the functional status of the tumours is provided (functionally active or not). 
GRS. According to the score from average tumour uptake and number of detected tumour sites, GRS was better than SRS in 13 patients (21.7\%) and equivalent images were obtained in 18 cases $(30.0 \%)$, while SRS performed better in $24(40.0 \%)$ of the patients (the rest of the patients was negative in SRS and GRS). Especially in carcinoids, GRS scored higher in $27.5 \%$ of the patients due to the detection of more tumour sites or higher uptake. In these patients, GRS showed a detection rate of tumour sites of $78.2 \%$ and SRS of $85.2 \%$. The complete results for all subgroups of tumour types are shown in Table 1, patient examples are shown in Figs 1-4.

$\kappa$-Statistics showed a high inter-rater reliability of 0.82 for SRS and 0.87 for GRS. Histological grading (high/intermediate/low differentiation) of the tumour was available in 36 patients with NET and Ki67 values in 32 . In 27 patients, both Ki67 and grading had been determined. Ki67 values and tumour grading were missing in patients whose primary pathology had not been done in our institution. There was no significant Pearson's correlation between the results of scintigraphy and the grade of tumour differentiation ( $r=0.028, P=0.873)$, but there was a weak inverse correlation of GRS and SRS both being positive and the rate of $\mathrm{Ki67}$ expression $(r=0.411, P=0.019)$. Therefore, no conclusion can be drawn that GRS is preferable as first-line imaging modality in a certain group of patients.

No correlations between the performance of GRS or SRS and the localisation of the primary (foregut, midgut and hindgut; data of 42 patients with known localisation of the primary, $r=-0.211, P=0.18$ ) or the functional status of the NET $(r=-0.004, P=0.98)$ was observed in the patients with gastrointestinal NET. The three patients with paragangliomas were all SRS positive, but only one was GRS-positive (Table 2).

\section{Discussion}

Our study demonstrates that GRS may provide additional information on patients with NET, especially if somatostatin uptake is missing. GRS detected additional tumour sites in $20 \%$ of all patients, half of which were negative in SRS. This indicates that minigastrin-binding $\mathrm{CCK}_{2}$ receptors can be present in patients with missing somatostatin receptor expression (i.e. expression of the subtypes binding Octreotide). Apart from imaging, an alternative to Octreotide could also be helpful when exploring the possibilities for optimization of peptide receptor radiotherapy (PRRT) by choosing the peptide with the more favourable tumour uptake, especially in patients showing low or missing Octreotide uptake.

\section{(a)}

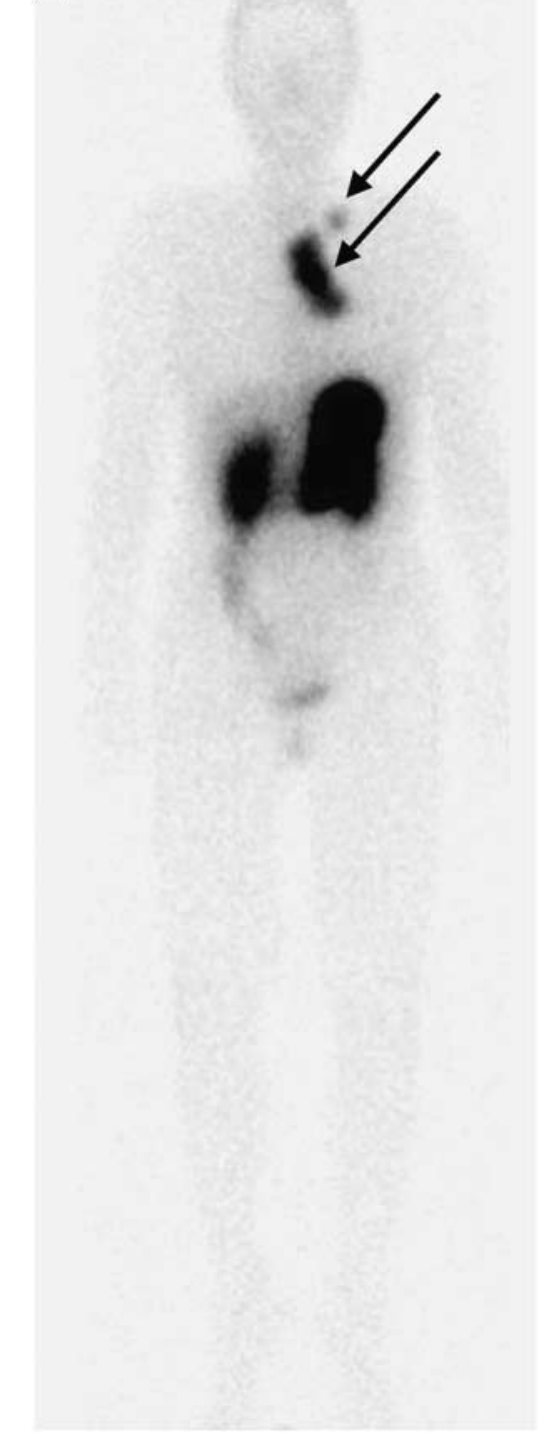

(b)

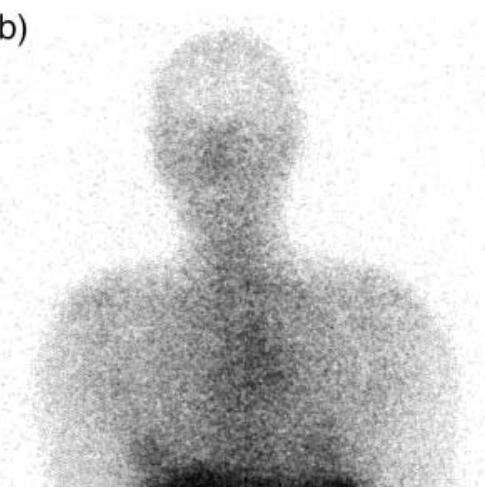

Figure 5 Anterior planar 24-h gastrin scan from a patient with small cell lung cancer. Uptake into the tumour and metastases is high (a), arrows while the somatostatin scan was negative (b), darker window, enlarged. 
Furthermore, it may be possible to increase the efficacy of PRRT using a cocktail of peptides targeting different receptors (Reubi \& Waser 2003).

Most of the radiopeptides introduced in clinical medicine or tested in clinical trials so far, are peptides also mainly targeting sstr2 and sstr5. Thus, their additional value in comparison to Octreotide with respect to targeting of new receptors is limited. The potential additional value of other non-sstr targeting peptides is abated by other properties, such as an unfavourable biodistribution with high background for example (Virgolini et al. 1994, 2002, Blum et al. 2000, Decristoforo et al. 2000, Hessenius et al. 2000, Reubi et al. 2000, Grewal et al. 2002, Janssen et al. 2002, De Visser et al. 2003, Gotthardt et al. 2004).

A possible advantage of gastrin scanning is that it can be performed in patients under therapy with longacting somatostatin analogues without restriction as Octreotide is not known to interact with the $\mathrm{CCK}_{2}$ receptor.

In the patient with a small cell lung cancer who had been excluded from the study because the diagnosis of a pulmonary carcinoid proved to be wrong, high uptake of gastrin in comparison with Octreotide was present (Fig. 5). GRS might thus also be of value in patients with small cell lung cancer (SCLC) where SRS does not perform well (Reisinger et al. 1998). This question should also be addressed in future studies.

In conclusion, GRS appears to add value in scintigraphic imaging in a subset of patients with NET. Gastrin scanning seems to be helpful if somatostatin scanning is negative, equivocal or showing low tumour uptake. Therefore, ${ }^{111}$ In-DTPA-DGlu ${ }^{1}$-minigastrin will not replace DPhe ${ }^{1}$-Octreotide in NET patients but should be positioned as an additional imaging modality in selected patients. Further studies have to be performed to address questions such as specificity of GRS in comparison with SRS or whether it may be of value in SCLC.

\section{References}

Bakker WH, Krenning EP, Reubi JC, Breeman WA, Setyono-Han B, de Jong M, Kooij PP, Bruns C, van Hagen PM, Marbach P et al. 1991 In vivo application of $\left[{ }^{111}\right.$ In-DTPA-D-PHE]-Octreotide for detection of somatostatin receptor-positive tumours in rats. Life Science 49 1593-1601.

Behe M, Kluge G, Becker W, Gotthardt M \& Behr TM 2005 Use of polyglutamic acids to reduce uptake of radiometallabeled minigastrin in the kidneys. Journal of Nuclear Medicine 46 1012-1015.

Behr TM, Jenner M, Béhé M, Angerstein C, Gratz S, Raue F \& Becker W 1999a Radiolabelled peptides for targeting of cholecystokinin-B/gastrin receptor expressing tumours: from preclinical development to initial clinical results. Journal of Nuclear Medicine 40 1029-1044.

Behr TM, Behe M, Angerstein C, Gratz S, Mach R, Hagemann L, Jenner N, Stiehler M, Frank-Raue K, Raue F \& Becker W 1999b Cholecystokinin-B/gastrin receptor binding peptides: preclinical development and evaluation of their diagnostic and therapeutic potential. Clinical Cancer Research 5 3124s-3138s.

Blum J, Handmaker H, Lister-James J \& Rinne N 2000 A multi-center trial with a somatostatin analog $99 \mathrm{mTc}-$ depreotide in the evaluation solitary pulmonary nodules. Chest 117 1232-1238.

De Visser M, Janssen PJJM, Srinivasan A, Reubi JC, Waser B, Erion JL, Schmidt MA, Krenning EP \& de Jong M 2003 Stabilised ${ }^{111}$ In-labelled DTPA- and DOTA-conjugated neurotensin analogues for imaging and therapy of exocrine pancreatic cancer. European Journal of Nuclear Medicine and Molecular Imaging 30 1134-1139.

Decristoforo C, Mather SJ, Cholewinski W, Donnemiller E, Riccabona G \& Moncayo R 2000 99mTc-EDDA/HYNICTOC: a new 99mTc-labelled radiopharamceutical for imaging somatostatin receptor-positive tumours: first clinical results and intra-patient comparison with

${ }^{111}$ In-labelled octreotide derivatives. European Journal of Nuclear Medicine 27 1318-1325.

Gotthardt M, Boermann OC, Behr TM, Béhé MP \& Oyen WJG 2004 Development and clinical application of peptide-based radiopharmaceuticals. Current Pharmaceutical Design 10 2951-2963.

Gotthardt M, Béhé M, Beuter D, Battmann A, Bauhofer A, Schurrat T, Schipper M, Pollum H, Oyen WJG, Behr TM 2006 Improved tumour detection by gastrin receptor scintigraphy in patients with metastasised medullary thyroid carcinoma. European Journal of Nuclear Medicine and Molecular Imaging (in press).

Grewal RK, Dadparvar S, Yu JQ, Babaria CJ, Cavanaugh T, Sherman M \& Jacobstein J 2002 Efficacy of 99mTcdepreotide scintigraphy in the evaluation of solitary pulmonary nodules. Cancer Journal 8 400-404.

Hessenius C, Bäder M, Meinhold H, Bohmig M, Faiss S, Reubi JC \& Wiedenmann B 2000 Vasoactive intestinal peptide receptor scintigraphy in patients with pancreatic adenocarcinomas or neuroendocrine tumours. European Journal of Nuclear Medicine 27 1684-1693.

Janssen ML, Oyen WJG, Dijkgraaf I, Massuger LF, Frielink C, Edwards DS, Rajopadhye M, Boonstra H, Corstens FH \& Boerman OC 2002 Tumour targeting with radiolabelled $\alpha v \beta 3$ integrin binding peptides in a nude mouse model. Cancer Research 62 6146-6151.

Juweid M, Sharkey RM, Behr T, Swayne LC, Rubin AD, Herskovic T, Hanley D, Markowitz A, Dunn R, Siegel J, Kamal T \& Goldenberg DM 1996 Improved detection of medullary thyroid cancer with radiolabelled antibodies to carcinoembryonic antigen. Journal of Clinical Oncology 14 1209-1217. 
Krenning EP, Kwekkeboom DJ, Bakker WH, Breeman WA, Kooij PP, Oei HY, van Hagen M, Postema PT, de Jong M, Reubi JC et al. 1993 Somatostatin receptor scintigraphy with $\left({ }^{111}\right.$ In-DTPA-D-Phe1)- and ( ${ }^{123}$ I-Tyr3)-Octreotide: the Rotterdam experience with more than 1000 patients. European Journal of Nuclear Medicine 20 716-731.

Lamberts SW, Bakker WH, Reubi JC \& Krenning EP 1990 Somatostatin-receptor imaging in the localization of endocrine tumours. New England Journal of Medicine 323 1246-1249.

Lebtahi R, Cadiot G, Sarda L, Daou D, Faraggi M, Petegnief Y, Mignon M \& le Guludec D 1997 Clinical impact of somatostatin receptor scintigraphy in the management of patients with neuroendocrine gastroenteropancreatic tumours. Journal of Nuclear Medicine 38 853-858.

Modlin IM \& Tang LH 1997 Approaches to the diagnosis of gut neuroendocrine tumours: the last word today. Gastroenterology 112 583-590.

Reisinger I, Bohuslavitzki KH, Brenner W, Braune S, Dittrich I, Geide A, Kettner B, Otto HJ, Schmidt S \& Munz DL 1998 Somatostatin receptor scintigraphy in small cell lung cancer: results of a multicenter study. Journal of Nuclear Medicine 39 224-227.

Reubi JC \& Waser B 2003 Concomitant expression of several peptide receptors in neuroendocrine tumours: molecular basis for in vivo multireceptor imaging. European Journal of Nuclear Medicine and Molecular Imaging 30 781-793.

Reubi JC, Schär JC, Waser B, Wenger S, Heppeler A, Schmitt JS \& Macke HR 2000 Affinity profiles for human somatostatin receptor subtypes sst1-sst5 of somatostatin radiotracers selected for scintigraphic and radiotherapeutic use. European Journal of Nuclear Medicine and Molecular Imaging 27 273-282.

Ricke J, Klose KJ, Mignon M, Öberg K \& Wiedenmann B 2001 Standardisation of imaing in neuroendocrine tumours: results of a European Delphi process. European Journal of Radiology 37 8-17.

Termanini B, Gibril F, Reynolds JC, Doppman JL, Chen CC, Stewart CA, Sutliff VE \& Jensen RT 1997 Value of somatostatin receptor scintigraphy: a prospective study in gastrinoma of its effect on clinical management. Gastroenterology 112 335-347.

Virgolini I, Raderer M, Kurtaran A, Angelberger P, Banyai S, Yang Q, Li S, Banyai M, Pidlich J, Niederle B, Scheithauer W \& Valent P 1994 Vasoactive intestinal peptide-receptor imaging for the localization of intestinal adenocarcinomas and endocrine tumours. New England Journal of Medicine 331 1116-1121.

Virgolini I, Britton K, Buscombe J, Moncayo R \& Paganelli G 2002 Riva $\mathrm{P}^{111} \mathrm{In}$ - and 90Y-DOTA-Lanreotide: results an implications of the MAURITIUS trial. Seminars in Nuclear Medicine 32 148-155. 